\title{
Review of: "The optimal emergency decision-making method with incomplete probabilistic information"
}

Jian Wu

Potential competing interests: No Conflict of interest

This article proposed a new emergency decision-making with probabilistic linguistic term. Its innovation is that it can deal with incomplete information. Its idea is interesting. But it has the following questions:

1 the introduction should refer and analysis more emergency decision-making methods, and then propose the research motivation.

2 Apart for fuzzy, the definitions are also used to express uncertainty: Twofold personalized feedback mechanism for social network consensus by uninorm interval trust propagation. IEEE Transactions on Cybernetics.Two stage feedback mechanism with different power structures for consensus in large-scale group decision-making. IEEE Transactions on Fuzzy Systems. The author should list a table to show these methods to deal with uncertainty

3 How to guarantee the proposed method of dealing with missing data is reasonable? Also, Can you make sure it has a mathematic of accurate solution of Model 1 o Eq(19)

4 In realistic emergency decision-making, it involves group decision makers, and then it usually requires group reach consensus before aggregation, such as: A bidirectional feedback mechanism for balancing group consensus and individual harmony in group decision making. Information Fusion. A dynamic feedback mechanism with attitudinal consensus threshold for minimum adjustment cost in group decision making. IEEE Transactions on Fuzzy Systems. 